\title{
Determination of Antibiotic Susceptibility of Rickettsia by the Plaque Assay Technique
}

\author{
JOSEPH E MCDADE \\ Biological Sciences Laboratories, Fort Detrick, Frederick, Maryland 21701
}

Received for publication 21 April 1969

Plaque formation by various rickettsiae was completely inhibited by commercial antibiotic dises impregnated with tetracycline, chloramphenicol, nitrofurantoin, and erythromycin; partial inhibition was observed around discs containing nalidixic acid and sulfisoxazole, but no inhibition was seen around discs containing cephalothin, ampicillin, oxacillin, kanamycin, polymyxin B, streptomycin, or penicillin.

A sensitive new plaque assay system for rickettsiae was recently reported (1). One of the advantages of a plaque system is that it is more versatile than the embryonated egg $L D_{50}$ assay. An adaptation reported here describes how commercial antibiotic sensitivity discs can be used in the plaque assay system to screen for antibiotic sensitivity of Rickettsia.

Stock suspensions of Rickettsia rickettsii Bitter Root (R1), R. akari MK, R. mooseri Wilmington,
R. prowazeki Madrid E, and R. prowazeki Breinl were prepared by a modification of the procedure of Weiss et al (2). Infected yolk sacs were homogenized in a blendor, partially purified by low- and high-speed centrifugation, suspended in sucrosephosphate, $p H$ 7.0, and stored at $-65 \mathrm{C}$.

The plaque assay procedure used in these studies is essentially the same procedure which was described eartier. Chick embryo primary cell monolayers $(24 \mathrm{hr})$ in plastic tissue culture flasks

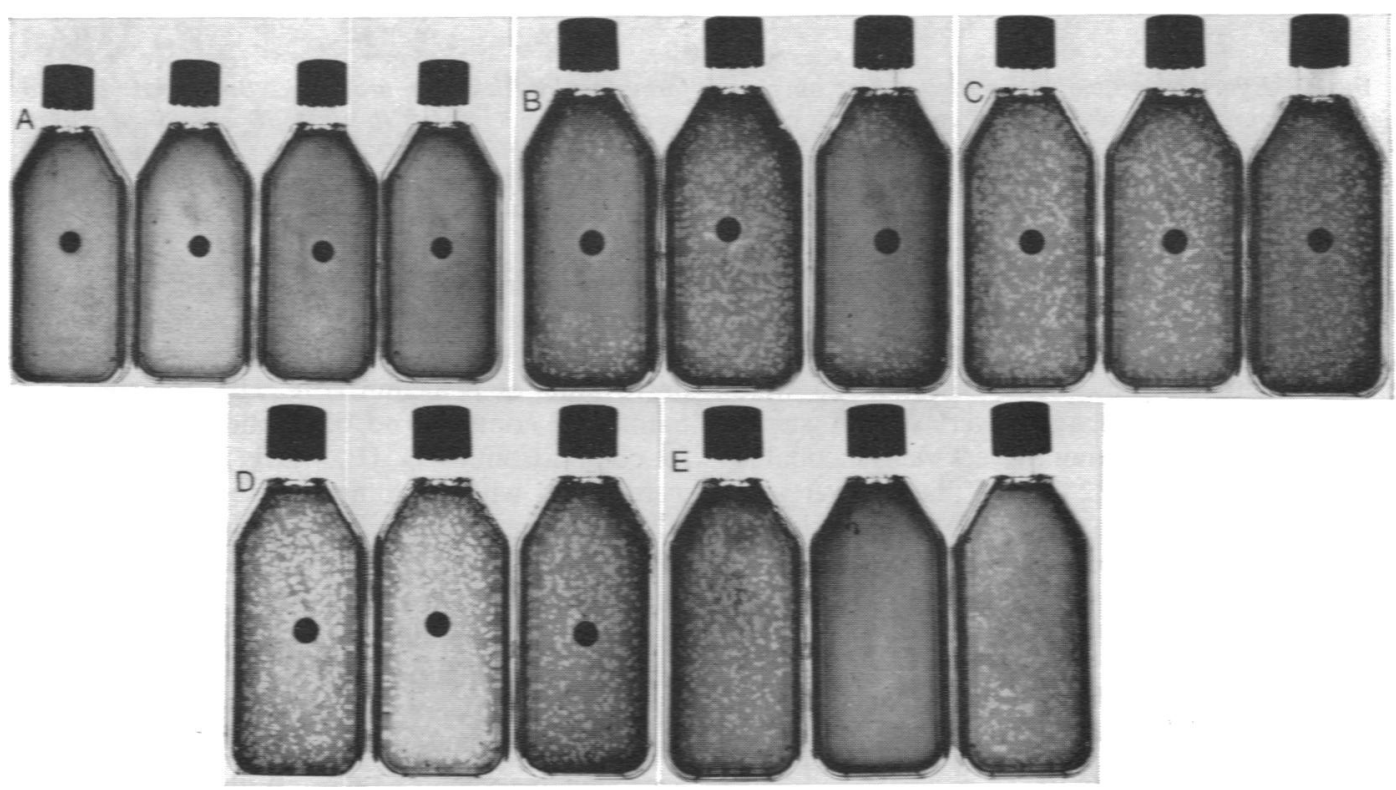

FIG. 1. Effect of antibiotic sensitivity discs on plaque formation by Rickettsia rickettsi Bitter Root. (A) Left to right, $30 \mu \mathrm{g}$ of tetracycline, $100 \mu \mathrm{g}$ of nitrofurantoin, $30 \mu \mathrm{g}$ of chlroamphenicol, $15 \mu \mathrm{g}$ of erythromycin; (Bleft to right, $30 \mu \mathrm{g}$ of nalidixic acid, 10 units of penicillin, $1.0 \mathrm{mg}$ of sulfisoxazole; (C) left to right, $30 \mu \mathrm{g}$ of ceph) alothin, $10 \mathrm{\mu g}$ of ampicillin, $1 \mathrm{\mu g}$ of oxacillin; (D) left to right, $5 \mathrm{\mu g}$ of kanamycin, 300 units of polymyxin $B, 10$ $\mu g$ of streptomycin; (E) left to right, infected, uninfected, and infected controls. 


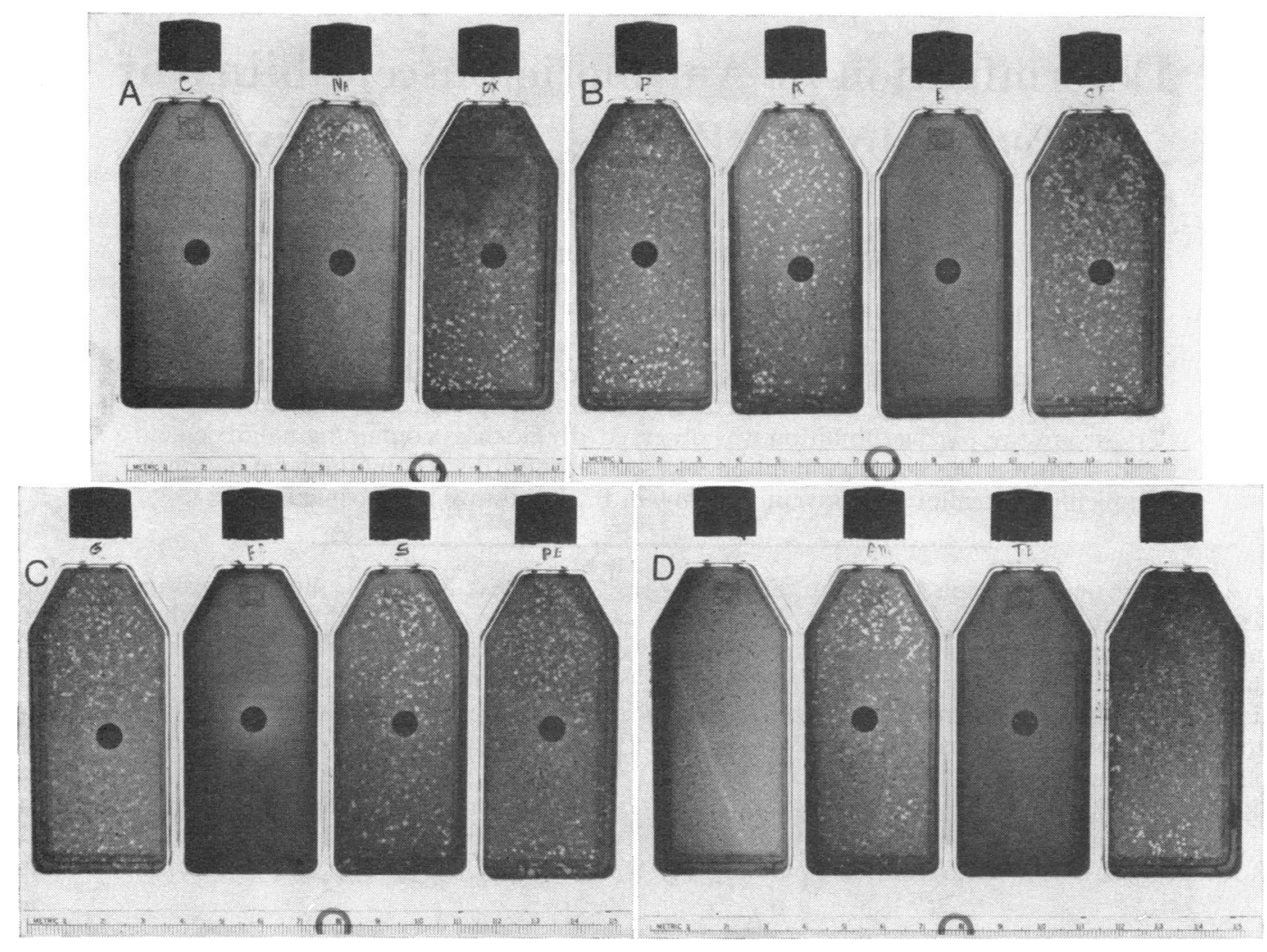

FIG. 2. Effect of antibiotic sensitivity discs on plaque formation by Rickettsia prowazeki Breinl. (A) Left to right, $30 \mu \mathrm{g}$ of chloramphenicol, $30 \mu \mathrm{g}$ of nalidixic acid, $1 \mathrm{\mu g}$ of oxacillin; (B) left to right, 10 units of penicillin, $5 \mu \mathrm{g}$ of kanamycin, $15 \mu \mathrm{g}$ of erythromycin, $30 \mu \mathrm{g}$ of cephalothin; (C) left to right, $1.0 \mathrm{mg}$ of sulfisoxazole, 100 $\mu g$ of nitrofurantoin, $10 \mu \mathrm{g}$ of streptomicin, 300 units of polymyxin $B ;(D)$ left to right, uninfected control, $10 \mu g$ of ampicillin, $30 \mu \mathrm{g}$ of tetracycline, infected control.

were infected with $0.1 \mathrm{ml}$ of a $10^{-3}$ dilution of stock suspensions and overlayed with medium 199 ( $5 \%$ calf serum) containing $0.5 \%$ agarose. Antibiotic sensitivity discs (Sensi-Discs; BBL) were then placed atop the agar overlay with sterile forceps. The infected monolayers were incubated in a closed system at $32 \mathrm{C}$ until plaques were observed on controls (no discs added). Monolayers were stained for photography by adding a second overlay containing medium 199 (no calf serum), $0.5 \%$ agarose, and neutral red at a final concentration of $0.01 \%$.

Figure 1 shows the effect of antibiotic sensitivity discs on $R$. rickettsii. Complete inhibition of plaque formation was observed with $30 \mu \mathrm{g}$ of tetracycline, $100 \mu \mathrm{g}$ of nitrofurantoin, $30 \mu \mathrm{g}$ of chloramphenicol, and $15 \mu \mathrm{g}$ of erythromycin (Fig. 1A); partial inhibition was observed with $30 \mu \mathrm{g}$ of nalidixic acid and $1.0 \mathrm{mg}$ sulfisoxazole (Fig. 1B); no inhibition was seen around discs containing 30 $\mu \mathrm{g}$ of cephalothin, $10 \mu \mathrm{g}$ of ampicillin, $1 \mu \mathrm{g}$ of oxacillin (Fig. 1C), $5 \mu \mathrm{g}$ of kanamycin, 300 units of polymyxin B, $10 \mu \mathrm{g}$ of streptomycin (Fig. 1D), or 10 units of penicillin (Fig. 1B). Infected and uninfected controls (no discs added) are shown for comparison in Fig. 1E. Nearly identical results were found with the other species of Rickettsia. All were completely inhibited by the same concentrations of tetracycline, nitrofurantoin, chloramphenicol, and erythromycin; each species was also partially inhibited by nalidixic acid, but no inhibition was apparent with the other antibiotics. Figure 2 shows the results obtained with $R$. prowazeki Breinl strain. The results of the other tests have been omitted because they were identical.

The susceptibility determinations reported here are in agreement with what is known about antibiotic susceptibility of rickettsiae. These results 
contain no new information on the susceptibility of rickettsiae to antibiotics, but they do indicate that this method may be more convenient and possibly more sensitive than egg assays for qualitative and quantitative determinations of antibiotic susceptibility in rickettsiae. This adaptation of the plaque technique should be useful in clinical and diagnostic microbiology and in the evalua- tion of new antibiotics, and may also provide a tool for genetic studies with rickettsiae.

\section{LTTERATURE GTTED}

1. Weinberg, E. H., J. R. Stakebake, and P. J. Gerone Plaque assay for Rickettsia ricketsii. J. Bacteriol. 98:398-402

2 Weies, E., H. B. Rees, Jr, and J. R. Hayes, 1967. Metabotic activity of purified suspensions of Ricketssia rickettsii. Nature (London) 213:1041-1022 